\title{
Remarks on Manifolds with Two-Sided Curvature Bounds
}

https://doi.org/10.1515/agms-2020-0122

Received January 21, 2021; accepted May 3, 2021

Abstract: We discuss folklore statements about distance functions in manifolds with two-sided bounded curvature. The topics include regularity, subsets of positive reach and the cut locus.

Keywords: Distance functions; cut locus; subsets of positive reach; harmonic coordinates; Alexandrov spaces MSC: 53C20, 53C21, 53C23

\section{Introduction}

\subsection{Distance functions in smooth Riemannian manifolds}

We discuss slightly generalized versions of some folklore results about distance functions $d_{A}$ to subsets $A$ of smooth Riemannian manifolds $M$. The results turn out to be local and are proved without any completeness assumptions. Our proofs do not involve Jacobi fields, but only basic facts about semiconcavity and semiconvexity of distance functions. Therefore, the statements generalize to the synthetic setting of manifolds with two-sided curvature bounds, as will be explained below.

The first statement is well-known in the complete situation, see, for instance, [26], [11], [15, Section 2].

Proposition 1.1. Let $M$ be a smooth Riemannian manifold. Let $A \subset M$ be a closed subset and let $f$ denote the distance function to the set $A$. Then $f$ is semiconcave in $M \backslash A$. The following conditions are equivalent for an open subset $O \subset M \backslash A$ :

1. $f$ is semiconvex in $O$.

2. $f$ is $\mathrm{e}^{1,1}$ in $O$.

3. $f$ is $\mathrm{e}^{1}$ in $O$.

4. For any $y \in O$, there exists at most one geodesic $\gamma_{y}:[0, \epsilon) \rightarrow$ M starting at y and parametrized by arclength, with the property $f \circ \gamma_{y}(t)=f(y)-t$, for all $t$ in $[0, \epsilon)$.

We assume some familiarity with the notions of semiconcave (semiconvex) functions and their gradient flows, [33], [3], [2]. Here and below, the notions of semiconcavity (convexity) and $e^{1,1}$ are local. For instance, we say that a function $f$ is $\mathrm{e}^{1,1}$ if it is $\mathrm{e}^{1}$ and the gradient $\nabla f$ is locally Lipschitz continuous.

For a closed subset $A$ of $M$, denote by $\operatorname{Reg}^{d_{A}}$ the set of points $x \in M \backslash A$, such that $d_{A}$ is $\mathrm{C}^{1}$ in a neighborhood of $x$. Thus, $\operatorname{Reg}^{d_{A}}$ is the maximal open set $O \subset M \backslash A$ which satisfies the equivalent conditions of Proposition 1.1. The closed subset $C L(A)=(M \backslash A) \backslash \operatorname{Reg}^{d_{A}}$ of $M \backslash A$ is called the cut locus of $A$ in $M$.

If $M$ is complete, then the geodesic $\gamma_{y}$ in (4) can be extended to a unique minimizing geodesic $\gamma_{y}$ : $\left[0, d_{A}(y)\right] \rightarrow M$ from $y$ to $A$. The endpoint of this geodesic is the unique projection point $\Pi^{A}(y)$ of $y$ on $A$. Moreover, the whole geodesic $\gamma_{y}\left(\left[0, d_{A}(y)\right)\right)$ is contained in $\operatorname{Reg}^{d_{A}}$.

^Corresponding Author: Vitali Kapovitch: University of Toronto, Toronto, Canada, E-mail: vkt@math.toronto.edu Alexander Lytchak: Mathematisches Institut, Universität Köln, Köln, Germany, E-mail: alytchak@math.uni-koeln.de 
If $M$ is complete and $A$ is a $e^{k}$ submanifold, for $k \geq 2$, then $d_{A}: \operatorname{Reg}^{d_{A}} \rightarrow \mathbb{R}$ can be expressed in terms of the normal exponential map of $A$ and turns out to be $\mathcal{C}^{k}$, see, for instance, [26, Prop. 4.3].

In the non-complete situation, none of the above statements need to hold, see Example 4.3.

The next result is known to specialists for some subsets in complete manifolds, [1], [4], [10], [9]. We include a short proof based on a general fact about gradient flows. Note, that the function $d_{A}$ is semiconcave on $M \backslash A$ and has a uniquely defined (local) gradient flow.

Proposition 1.2. Let $A$ be a closed subset of a Riemannian manifold $M$. Then the cut locus $C L(A) \subset M \backslash A$ of $A$ is invariant under the gradient flow $\Phi$ of the distance function $d_{A}$.

A natural generalization of the above result and its proof is valid in Alexandrov spaces, [3, Prop. 14.1.5]. As an application of Proposition 1.2 one can derive a very short proof of the nice geometric observation [15, Theorem 6.1], see Corollary 4.2 below.

Finally, we address (again essentially well-known to specialists) properties of subsets of positive reach, defined and investigated by Federer in Euclidean spaces, [13], and by Bangert and Kleinjohann in Riemannian manifolds, [6], [18]; see also [25], [23], [34].

Recall that a closed subset $A$ in a Riemannian manifold is said to have positive reach, if $A$ has a neighborhood $O$ such that the foot point projection $\Pi^{A}$ is uniquely defined on $O$. As has been shown by Federer and Bangert, the notion only depends on the underlying smooth structure and not on the Riemannian metric. The following result is essentially contained in [18], [6], [25].

Proposition 1.3. For a closed subset $A$ of a smooth Riemannian manifold $M$, the following are equivalent:

1. The subset $A$ is of positive reach.

2. There is an open neighborhood $O$ of $A$ such that the distance function $d_{A}$ is $\mathrm{C}^{1,1}$ in $O \backslash A$.

3. The function $d_{A}$ is semiconvex on a neighborhood $O$ of $A$.

See [6] and [25] for other characterizations.

Any subset of positive reach $A \subset M$ has a well-defined tangent cone $T_{x} A$ at every point $x \in A$. This tangent cone is a convex cone in $T_{X} M$, [13, Theorem 4.8]. The normal cone $T_{X}^{\perp} A$ is the convex cone of all vectors in $T_{x} M$ enclosing angles at least $\frac{\pi}{2}$ with all vectors in $T_{x} A$.

For the following folklore statement about subsets of positive reach we could not find appropriate references. In the Euclidean case the result is contained in [13].

Proposition 1.4. Let $A \subset M$ be a subset of positive reach. Then for any $x \in A$ and unit $h \in T_{x}^{\perp} A$ the geodesic $\gamma^{h}$ starting at $x$ in the direction of $h$ satisfies $d_{A}\left(\gamma^{h}(s)\right)=s$, for all $s>0$ such that $\gamma^{h}([0, s])$ is contained in the open subset $O$ from Proposition 1.3.

\subsection{Manifolds with two-sided curvature bounds}

Here we discuss some basic observations about non-smooth Riemannian manifolds which have two-sided curvature bounds in the sense of Alexandrov and the extensions of the above results to this setting. These results have been applied in [17]. Readers only interested in the smooth situation can skip this part of the introduction together with Section 6.

Manifolds with two-sided curvature bounds in the sense of Alexandrov appear in one of the most prominent examples of Gromov-Hausdorff convergence. Namely, the class of such compact manifolds with uniform bounds on injectivity radius, diameter and curvature is compact with respect to the Gromov-Hausdorff convergence, [8], [14], [31]. It provides a natural compactification of the corresponding class of smooth Riemannian manifolds. Moreover, all manifolds with two-sided curvature bounds in the sense of Alexandrov turn out to have a rather regular analytic structure and to admit a smoothing, as has been proved by Nikolaev in a series of papers. A good readable summary of these results of Nikolaev and related statements on the 
structure of such manifolds has appeared in [8]. Results and ideas of Nikolaev motivated many theorems in the theory of Alexandrov spaces with one-sided curvature bounds, [7], [16], [30], [29], [32], [19].

We will assume some familiarity with the theory of Alexandrov spaces, and refer the non-familiar reader to [3]. The most appropriate setting for our local results is the following one.

Definition 1.5. A locally compact length metric space $X$ has two-sided bounded curvature if for any point $x \in X$ there exists a compact convex neighborhood $U$ and some $K>0$ such that $U$ is an Alexandrov space of curvature $\geq-K$ and $a C A T(K)$ space.

A space $X$ with two-sided bounded curvature is topologically a manifold $M$ with boundary $\partial M$. Moreover, $M \backslash \partial M$ is convex in $M$, [8], cf. [16]. We restrict the attention to the case $\partial M=\emptyset$.

By a manifold with two-sided curvature bounds we will denote a space as in the above Definition 1.5 which, in addition, is homeomorphic to a manifold without boundary.

Any manifold $M$ with two-sided curvature bounds admits a natural atlas of distance coordinates, see [8] and Section 3 below. The distance in $M$ is defined by a $e^{0,1}$ Riemannian metric $g$ in this atlas. Moreover, the $\mathfrak{e}^{1,1}$-smoothness of the atlas and the $e^{0,1}$-smoothness of the Riemannian metric is optimal, as can be observed in the manifold $M$ arising from the gluing of a flat cylinder and a hemisphere. Results of this type with weaker conclusions have been obtained for distance coordinates under one-sided curvature bounds, [30], [29], [19].

On any $\mathcal{C}^{1,1}$ manifold $M$ with a Lipschitz continuous Riemannian metric, any harmonic function is $\mathrm{C}^{1, \alpha}$, for all $\alpha<1$, [38, III, Chapter 9] and there exist harmonic coordinates around any point, [38, III, Chapter 9]. The atlas of harmonic coordinates is of class $\mathcal{C}^{2, \alpha},[37$, p. 689] and the distance is defined by a metric of class $e^{\alpha}$ in these coordinates. One of the central results of Nikolaev's theory, see [8], is that for any manifold $M$ with two-sided curvature bounds, the harmonic atlas is of class $\mathcal{C}^{3, \alpha}$, for any $\alpha<1$, and the Riemannian metric in this atlas is of class $\mathcal{C}^{1, \alpha}$, for any $\alpha<1$.

In a general $\mathrm{e}^{1,1}$-manifold with a $\mathrm{C}^{0,1}$ Riemannian metric a harmonic function does not need to be of class $\mathrm{e}^{1,1}$, cf. [37, p. 693] and Problem 1.9 below. Our first observation is that such a loss of smoothness cannot happen on a manifold with two-sided bounded curvature.

Proposition 1.6. Let $M$ be a manifold with two-sided bounded curvature. Then any harmonic function on an open subset $U$ in $M$ is of class $\mathrm{C}^{1,1}$ in distance coordinates. Thus, any transformation from distance to harmonic coordinates is of class $\mathrm{e}^{1,1}$.

Thus, the $\mathrm{e}^{1,1}$ atlas of distance coordinates can be assumed to include all harmonic coordinates as well. From now on all statements will refer to this $\mathrm{C}^{1,1}$ atlas. The following result might be folklore knowledge:

Proposition 1.7. Let $M$ be a manifold with two-sided curvature bounds and let $N \subset M$ be a $\mathrm{C}^{1,1}$ submanifold. Then $N$ with its intrinsic metric is a manifold with two-sided curvature bounds. Moreover, $N$ has positive reach in $M$.

This result follows from the Gauß equation and another central result of Nikolaev's theory, stating that manifolds with two-sided curvature bounds are exactly the limit spaces (in a precise local sense) of smooth Riemannian manifolds with uniform bounds on sectional curvature.

The final statement discussed in the introduction is that all results about distance functions in smooth Riemannian manifolds are valid in this more general setting:

Theorem 1.8. The statements of Proposition 1.1, Proposition 1.2 and Proposition 1.3 are valid for any closed subset $A$ of any manifold $M$ with two-sided bounded curvature. The differentiability in Propositions 1.1, 1.3 is considered with respect to distance coordinates.

In fact, Propositions 1.1, 1.2 and 1.3 are directly proven in the metric setting of manifolds with two-sided curvature bounds. 


\subsection{A few questions}

We would like to finish the introduction with a few open questions about manifolds with two-sided curvature bounds.

Problem 1.9. Does there exist a Riemannian metric of class $\mathrm{e}^{0,1}$ and a harmonic function with respect to this metric, which is not $\mathrm{e}^{1,1}$ ?

It is possible that a positive answer to this problem might be obtained following the ideas in the examples discussed in [37, p. 693].

The second part of the next problem is motivated by Proposition 1.7. It should be compared with Nash's embedding theorem for Riemannian manifolds of higher regularity, see [5].

Problem 1.10. Let $M$ be a manifold with two-sided curvature bounds in the sense of Alexandrov. Do there exist coordinates on $M$ in which the distance is defined by a Riemannian metric of class $\mathrm{C}^{1,1}$ ? Can $M$ be lengthpreserving embedded as a $\mathrm{e}^{1,1}$ submanifold in a Euclidean space?

We would like to mention that the folklore argument providing a negative answer to the first question above, for instance, [31], is not correct. Indeed, [31] provides a surface with two-sided curvature bounds such that in harmonic coordinates the Riemannian metric is not of class $\mathcal{C}^{1,1}$. Then the proof invokes the statement of [12], [37] that the smoothness of the metric is optimal in harmonic coordinates. However, this elliptic regularity statement is not covered by [12] (since elliptic regularity does not work well for Lipschitz functions). Indeed, the following result has appeared in [37, Example 2] and provides a counter-example to the folklore proof. There exists a Riemannian metric of class $\mathcal{C}^{2}$ on $\mathbb{R}^{2}$ such that in any harmonic coordinates the metric is not $\mathrm{e}^{1,1}$.

The second question concerns the existence of a canonical smoothing of manifolds with two-sided curvature bounds. While the existence part in the next question is a direct consequence of the approximation results of Nikolaev and [36], the uniqueness is more subtle:

Problem 1.11. Let $M$ be a complete manifold with two-sided bounded curvature. If the curvature bounds can be chosen uniformly on $M$ then there exists a unique Ricci flow coming out of the manifold $M$.

The final problem we would like to mention is due to the following fact. The theory of Nikolaev is scattered through several works and some of them are not easy to read (and to find). Thus, we formulate:

Problem 1.12. Find a streamlined proof of Nikolaev's result on smoothings of manifolds with two-sided curvature bounds, [28], [27].

\subsection{Structure of the paper}

In Section 2, we recall basics on semiconcave functions and their gradient flows and verify a local version of Proposition 1.2. In Section 3, we recall basic facts about distance coordinates. In Section 4, we prove Proposition 1.1 and 1.2. In Section 5, we prove the stated results about subsets of positive reach, Propositions 1.3, 1.4. Finally, in Section 6, we prove Proposition 1.6 and Proposition 1.7. 


\section{Semiconcavity and gradient flows}

\subsection{Notation}

Distance will be denote by $d$. The distance function from a subset $A$ of a space $X$ will be denote by $d_{A}$. By definition, this is a 1-Lipschitz function. A geodesic will denote an isometric embedding $\gamma: I \rightarrow X$ of an interval. Thus, our geodesics are always parametrized by arclength and globally minimizing.

\subsection{Special neighborhood}

Let $M$ be a manifold with two-sided curvature bounds. For any point $x \in M$, we find a compact neighborhood $U$ of $x$ as in Definition 1.5. Restricting the neighborhood and using convexity of small balls in $C A T(K)$ spaces we may assume that the neighborhood $U=U_{x}$ has the following form.

The set $U_{x}$ is the closed ball of radius $r_{x}$ around $x$ and it is homeomorphic to a Euclidean ball, [8, Theorem 12.1]. Any pair of points in $U_{x}$ is connected by a unique geodesic in $U_{x}$. For some $K_{x}>0$, the space $U_{x}$ is $\operatorname{CAT}\left(K_{x}\right)$ and an Alexandrov space of curvature $\geq-K_{x}$, moreover, $\epsilon_{x}:=r_{x} \cdot K_{x}<<1$. Any geodesic in $U_{x}$ extends to a geodesic starting and ending on the distance sphere $\partial U_{x}$, [8, Prop. 8.3].

\subsection{Semiconvexity, semiconcavity and gradient flows}

A locally Lipschitz function $f$ on an open subset $O$ of $M$ is $C$-concave, respectively $C$-convex, if for any geodesic $\gamma: I \rightarrow O$ the function $f \circ \gamma(t)-\frac{C}{2} t^{2}$ is concave, respectively convex, on $I$.

A function $f$ is $C$-concave if and only if for all pairs of points $p_{1}, p_{2} \in O$ which are sufficiently close to each other and any midpoint $m$ between $p_{1}$ and $p_{2}$, we have

$$
f(m) \geq \frac{1}{2}\left(f\left(p_{1}\right)+f\left(p_{2}\right)\right)-\frac{C}{8} \cdot d^{2}\left(p_{1}, p_{2}\right) .
$$

The function $f: O \rightarrow \mathbb{R}$ is semiconcave (semiconvex) if for any $x \in O$ there is some $C \in \mathbb{R}$, such that the restriction of $f$ to some neighborhood of $x$ in $O$ is $C$-concave ( $C$-convex).

If $f: O \rightarrow \mathbb{R}$ is semiconcave and $h: O \rightarrow \mathbb{R}$ is continuous, we say that the function $f$ is $h$-concave, if for any $x \in O$ and any $\epsilon>0$, there exists a neighborhood $O_{\epsilon}$ of $x$ such that $f$ is $(h(x)+\epsilon)$-concave in $O_{\epsilon}$.

This is equivalent to the requirement that, for any geodesic $\gamma: I \rightarrow O$, we have $(f \circ \gamma)^{\prime \prime} \leq h \circ \gamma$ on $I$ in the sense of distributions.

Since the notion of semiconcavity and of gradient curves and flows of semiconcave functions is local, the whole theory of gradient flows in Alexandrov spaces, [3], [33], applies to the present situation.

For any semiconcave function $f: O \rightarrow \mathbb{R}$ and every $x \in O$ there exists a unique vector $\nabla_{x} f \in T_{x} O$, the gradient of $f$ at $x$. Moreover, there exists a unique maximal curve $\eta_{x}:[0, a) \rightarrow O$ with some $a \in(0, \infty)$, the gradient curve of $f$, which starts in $x$ and satisfies

$$
\eta_{x}^{\prime}(t)=\nabla_{\eta_{x}(t)} f \text { and }\left(f \circ \eta_{x}\right)^{\prime}(t)=\left|\nabla_{\eta_{x}(t)} f\right|^{2},
$$

for all $t \in[0, a)$. Furthermore, if $a<\infty$ then $\eta_{x}([0, a))$ is not contained in any compactum in $O$. Finally, the $\operatorname{map}(x, t) \rightarrow \Phi(t, x):=\eta_{x}(t)$ is a local flow defined on a neighborhood of $O \times\{0\} \subset O \times[0, \infty)$ and it is locally Lipschitz continuous, [33].

A point $x \in O$ is critical for $f$ if $\nabla_{x} f=0$. In this case $\eta_{x}$ is the stationary curve $\eta_{x}(t)=x$. On the other hand, if the curve $\eta_{x}$ does not contain critical points of $f$, it has a unique parametrization $\tilde{\eta}_{x}:[0, \tilde{a}) \rightarrow O$ by arclength.

The following is a not very well-known but fundamental observation. The first statement is exactly [3, Theorem 14.1.3]; the second one follows from the first by localization: 
Lemma 2.1. Let $f: O \rightarrow \mathbb{R}$ be $C$-concave. Then for the arclength reparametrization $\tilde{\eta}_{x}$ of any gradient curve $\eta_{x}$ of $f$ in $O$, the composition $f \circ \tilde{\eta}_{x}:[0, a) \rightarrow \mathbb{R}$ is $C$-concave.

If $f$ is $h$-concave for a continuous function $h: O \rightarrow \mathbb{R}$ then $f \circ \tilde{\eta}_{x}$ is $h \circ \tilde{\eta}_{x}$-concave on $[0, a)$.

\subsection{Distance functions in special neighborhoods}

Let $U \subset M$ be a special neighborhood of some point as in Subsection 2.2. Thus, $U$ is convex, compact, $C A T(K)$ and it is an Alexandrov space of curvature $\geq-K$, for some $K>0$.

Then, for any point $x \in U$ the distance function $f=d_{x}$ is convex in $U$. Moreover, $f$ is also semiconcave on $U \backslash\{x\}$. More precisely, on the set $O$ of points $y \in U$ with $d(x, y)>\delta$, the function $f$ is $C$-concave, for some $C=C(K, \delta)$.

Since an infimum of $C$-concave functions is $C$-concave, for any subset $B \subset U$ the distance function $d_{B}$ is $C(K, \delta)$ concave on the set of all points $y \in U$ with $d_{B}(y)>\delta$.

The function $f=d_{B}$ is 1-Lipschitz, thus $\left|\nabla_{y} f\right| \leq 1$ for all $y \in U \backslash B$. By the first variation formula, $\left|\nabla_{y} f\right|=1$ if and only if $y$ is connected with $B$ by a unique shortest geodesic.

The conclusion of Proposition 1.2 will follow from the next Lemma, cf. [3, Prop. 14.1.5], [1, Theorem 4.5]:

Lemma 2.2. Let $B \subset U$ be any closed subset in a special neighborhood $U$ as above. Let $y \in U \backslash B$ be arbitrary and let $\eta_{y}:[0, a) \rightarrow U$ be the gradient curve in $U$ of the distance function $f=d_{B}$ starting at $y$. If $\left|\nabla_{y} f\right|<1$ then $\left|\nabla_{z} f\right|<1$, for all $z$ on the gradient curve $\eta_{y}$.

Proof. Rescaling the space, we may assume that the lower curvature bound on $U$ equals -1 . Then, for the distance function $F=d_{p}$ to any $p \in U$ the composition $\hat{F}=\cosh \circ F$ is $\hat{F}$-concave on $U$, [3, Theorem 7.4.1]. Hence, also for the infimum $f=d_{B}$ of distance functions to points, the composition $\hat{f}:=\cosh \circ f$ is $\hat{f}$-concave on $U$.

The gradient curves of $f$ and $\hat{f}$ on $U \backslash B$ coincide up to parametrizations, [3, Theorem 11.4.4]. Thus, the arclength reparametrization $\tilde{\eta}_{y}$ of $\eta_{y}$ is also the arclength parametrization of the gradient curve of $\hat{f}$. From Lemma 2.1, we deduce that $\hat{f} \circ \tilde{\eta}_{y}$ is $\hat{f} \circ \tilde{\eta}_{y}$-concave on the interval of definition $[0, b]$ of $\tilde{\eta}_{y}$ from $y$ to $z$.

Define $h:[0, b] \rightarrow \mathbb{R}$ as $h(t)=f \circ \tilde{\eta}_{y}(t)$. Then $h$ is an increasing, 1-Lipschitz, semiconcave function; we have $h^{\prime}(0)<1$ and the composition $\hat{h}:=\cosh \circ h$ is $\hat{h}$-concave. We only need to verify $h^{\prime}(b)<1$.

Assume, by contrary that $h^{\prime}(b)=1$. Consider the linear function $h_{0}(t)=t+(h(b)-b)$. Then

$$
h_{0}(b)=h(b) ; h_{0}^{\prime}(b)=h^{\prime}(b) ;\left(\cosh \circ h_{0}\right)^{\prime \prime}=\cosh \circ h_{0} .
$$

By comparison, cf. [3, Theorem 4.5.3], we deduce

$$
\cosh \circ h(t) \leq \cosh \circ h_{0}(t),
$$

for all $t \in[0, b]$. Hence $h(t) \leq h_{0}(t)$ for all $t$. However, $h$ is 1-Lipschitz. Thus, $h(t)=h_{0}(t)$ for all $t \in[0, b]$. This implies $h^{\prime}(0)=1$ in contradiction to our assumption. This finishes the proof.

\subsection{Distance functions to remote subsets}

Let $M$ be a manifold with two-sided curvature bounds (possibly non-complete and without uniform bounds on curvature), as in Subsection 1.2. Let $A$ be a closed subset of $M$ and consider the distance function $f=d_{A}$ to $A$.

Consider an arbitrary point $x \in M \backslash A$ and a special neighborhood $U=U_{x}=\bar{B}_{r_{x}}(x)$ as in Subsection 2.2. Making $r_{X}$ smaller, we may assume that $U$ is disjoint from $A$.

Let $\tilde{U}$ be a smaller ball of radius $r<\frac{1}{3} \cdot r_{x}$ around $x$. Set $s=f(x)$ and denote by $B$ the compact subset $B=f^{-1}(s-2 r) \cap U$. By compactness and convexity of $U$, for any $y \in \tilde{U}$, we find at least one footpoint $\hat{y}$ of $y$ 
on $B$, thus $d(y, \hat{y})=d_{B}(y)$. By the triangle inequality,

$$
f(y) \leq f(\hat{y})+d(y, \hat{y})=(s-2 r)+d_{B}(y) .
$$

On the other hand, any curve $\eta$ from $A$ to $y$ must contain points $z$ with $f(z)=s-2 r$. If $z$ is not in $U$ then the length of $\eta$ is at least $s+r$. Thus, any such $\eta$ with length less than $s+r$, contains points on $B$. Thus by the triangle inequality we deduce the equality

$$
d_{A}=f=(s-2 r)+d_{B}
$$

on $\tilde{U}$. Since $d_{B}$ is semiconcave on $\tilde{U}$, so is $f$, moreover, the gradient curves of $f$ and $d_{B}$ coincide in $\tilde{U}$.

Now we arrive at the following results (also valid in all Alexandrov spaces and their localized version, Alexandrov regions, [20]).

Corollary 2.3. Let $A$ be a closed subset in a manifold $M$ with two-sided curvature bounds. Then, the distance function $f=d_{A}$ is semiconcave on $M \backslash A$. For any point $x \in M \backslash A$ with $\left|\nabla_{x} f\right|<1$ we have $\left|\nabla_{y} f\right|<1$ for all $y$ on the gradient curve $\eta_{x}$.

Proof. The semiconcavity condition is local and has been verified above in the neighborhood of any $x \in M \backslash A$.

In order to see the second statement, we consider the compact part $\eta_{x}:[0, b] \rightarrow M$ of the gradient curve $\eta_{x}$ between $x$ and $y$. Assuming the contrary, and using that $f \circ \tilde{\eta}_{x}$ is semiconcave, we find a smallest $t \in(0, b]$ such that $\left|\nabla_{\eta_{x}(t)} f\right|=1$.

Now we find a small special neighborhood $U$ of $z=\eta_{x}(t)$, identify on a smaller neighborhood $\tilde{U}$ (up to an additive constant) $f$ with $d_{B}$ for a closed subset $B \subset U$ and derive a contradiction to Lemma 2.2.

\section{Coordinates}

\subsection{Distance coordinates}

Let $U \subset M$ be a special neighborhood of a point $x$ in a manifold $M$ with two-sided curvature bounds.

For any $x \in U$ the function $d_{x}$ is convex in $U$. Moreover, $d_{x}$ is $C(K, \delta)$ concave on the set of points $y$ in $U$ with $d(x, y)>\delta$.

For any $p$ in the interior of $U$, consider any points $p_{1}, \ldots, p_{n}$ in $U$ such that the starting directions of the geodesics $p p_{i}$ are almost orthogonal at $p$, (see [8, Theorem 13.2]). Then the map $F: U \rightarrow \mathbb{R}^{n}$ with coordinates $f_{i}:=d_{p_{i}}$ is a biLipschitz map $F: O \rightarrow \tilde{O}$ from an open ball $O$ around $p$ onto an open subset of $\mathbb{R}^{n}$. (This is even true for any Alexandrov space, [7]).

Any such map $F: O \rightarrow \tilde{O}$ is called a distance chart on $M$. The distance charts define a $\mathrm{C}^{1,1}$ atlas on $M$ and the distance on $M$ is given by a Riemannian metric of class ${ }^{0,1}$ with respect to this atlas, [8, Theorem 13.2].

\subsection{Distance charts and semiconcavity}

The following observation can also be used to obtain a shorter alternative proof of [8, Theorem 13.2], using the observation that a homeomorphism between open subsets of $\mathbb{R}^{n}$ is $\mathcal{C}^{1,1}$ if and only if it preserves the class of semiconcave functions.

Lemma 3.1. Let $F: O \rightarrow \tilde{O} \subset \mathbb{R}^{n}$ be a distance chart in a manifold with two-sided curvature bounds. Then a function $f: \tilde{O} \rightarrow \mathbb{R}$ is semiconcave if and only if $f \circ F$ is semiconcave on $O$.

Proof. From the biLipschitz property of $F$, and semiconcavity and semiconvexity of the coordinates $f_{i}$, we deduce the following. For any geodesic $\gamma$ in $O$ connecting $q_{1}$ and $q_{2}$ and having $m$ as its midpoint the distance 
between $F(m)$ and the midpoint $\bar{m}=\frac{1}{2}\left(F\left(q_{1}\right)+F\left(q_{2}\right)\right)$ in $\mathbb{R}^{n}$ between $F\left(q_{i}\right)$, we have

$$
d(F(m), \bar{m}) \leq C \cdot d^{2}\left(q_{1}, q_{2}\right) .
$$

Here the constant $C$ depends only on the biLipschitz constant of $F$ and the curvature bounds.

Since $F$ is biLipschitz, $F^{-1}$ sends midpoints in $\tilde{O}$ to "almost midpoints" in $O$ in the same sense as above. Now the equivalence of semiconcavity of $f$ and $f \circ F$ follows after applying (2.1).

\section{Main results}

\subsection{General distance functions}

We are going to prove the following slight generalization of Proposition 1.1

Proposition 4.1. Let $M$ be a manifold with two-sided curvature bounds. Let $A \subset M$ be a closed subset and $f=d_{A}$. Then the following conditions are equivalent for an open subset $O \subset M \backslash A$ :

1. $f$ is semiconvex in $O$.

2. $f$ is $\mathrm{e}^{1,1}$ in $O$.

3. $f$ is $\mathrm{e}^{1}$ in $O$.

4. For all $x \in O$ we have $\left|\nabla_{x} f\right|=1$

5. For any $x \in O$, there exists at most one geodesic $\gamma_{x}:[0, \epsilon) \rightarrow M$ starting at $x$, with $f \circ \gamma_{x}(t)=f(x)-t$, for all $t$ in $[0, \epsilon)$.

Proof. All statements are local on $O$. We may fix $p \in O$ and consider a special neighborhood $U$ of $p$ in $M$. Furthermore, in Subsection 2.5, we have found a closed subset $B$ in $U$ and a smaller neighborhood $O_{0}$ of $p$ in $O$, such that on $O_{0}$ the function $f$ coincides with $d_{B}$ up to an additive constant. Thus, we may assume without loss of generality, that $A=B \subset U$ and $O=O_{0}$. Making $O$ smaller, if needed, we may assume that the open neighborhood $O$ is a coordinate chart. Thus, on $O$ the notion of semiconcavity is the same with respect to the metric structure and to the coordinate chart.

The semiconcavity of $f$ has been verified in Corollary 2.3. Since on open subsets of $\mathbb{R}^{n}$ a function is $\mathrm{e}^{1,1}$ if and only if it is semiconcave and semiconvex, the properties (1) and (2) are equivalent.

Clearly, (2) implies (3).

For any $x \in O$, we find, by compactness, at least one shortest geodesic $\gamma_{x}:[0, f(x)] \rightarrow U$ from $x$ to $A=B$. Then $\left(f \circ \gamma_{x}\right)^{\prime}=-1$ on $[0, f(x))$. This shows, that for all $t \in(0, f(x))$, we have $\left|\nabla_{\gamma_{x}(t)} f\right|=1$. If $f$ is $\mathcal{C}^{1}$ it also implies $\left|\nabla_{x} f\right|=1$. Hence, (3) implies (4).

By the first formula of variation, $\left|\nabla_{x} f\right|=1$ if and only $x$ is connected with $B$ by exactly one shortest geodesic $\gamma_{x}$. Note that for any such geodesic $f \circ \gamma_{x}(t)=f(x)-t$ for all $t \in[0, f(x)]$. Moreover, for any geodesic, $\tilde{\gamma}_{x}:[0, \epsilon) \rightarrow U$ satisfying the above equality for all $t \in[0, \epsilon)$, the unique extension of $\tilde{\gamma}_{x}$ to length $f(x)$ ends on $B$. This shows the equivalence of (4) and (5).

It remains to show that (4) implies (1). In this case, gradient lines $\eta_{x}$ of $f$ in $O$ are parametrized by arclength and $f \circ \eta_{x}$ has everywhere velocity 1 . This implies that any gradient line $\eta_{x}$ in $O$ is an (as always minimizing) geodesic.

Fix $x \in O$ and consider $\delta$ such that the ball of radius $3 \delta$ around $x$ is contained in $O$. Consider $C=C(K, \delta)$, the semiconcavity constant of distance functions in $O$ at distance $\geq \delta$, as in Subsection 2.4. We claim that $f$ is $-C$-convex on the ball $W$ of radius $\delta$ around $x$.

Indeed, consider points $q_{1}, q_{2} \in W$ and their midpoint $m$. Set $z=\eta_{m}(2 \delta)$. Then, for $i=1,2$,

$$
f(z)=f(m)+2 \delta=f(m)+d(m, z) \leq f\left(q_{i}\right)+d\left(q_{i}, z\right),
$$

due to the triangle inequality. This implies

$$
f(m) \leq \frac{1}{2}\left(f\left(q_{1}\right)+f\left(q_{2}\right)\right)+\left(\frac{1}{2}\left(d\left(q_{1}, z\right)+d\left(q_{2}, z\right)\right)-d(m, z)\right) \leq
$$




$$
\leq \frac{1}{2}\left(f\left(q_{1}\right)+f\left(q_{2}\right)\right)+\frac{C}{8} \cdot d^{2}\left(q_{1}, q_{2}\right) .
$$

This implies the claimed semiconvexity of $f$.

\subsection{Cut locus}

As a combination of previous results we now obtain

Proof of Proposition 1.2. As before set $f=d_{A}$. Due to Proposition 4.1, the cut locus $C L(A)$ of $A$ is exactly the closure $C L(A)=\bar{X}$ of

$$
X=\left\{x \in M \backslash A:\left|\nabla_{x} f\right|<1\right\} .
$$

As we have seen in Corollary 2.3, the set $X$ is invariant under the gradient flow of $f$. By continuity of the gradient flow, the closure $\bar{C}=C L(A)$ is invariant under the gradient flow as well.

As a consequence we deduce the following observation from [15]:

Corollary 4.2. Let $M$ be a complete Riemannian manifold. Let $A$ be a closed subset of $M$ and let $X=C L(A) \subset$ $M \backslash A$ be the cut locus of $A$. Assume that the distance function $d_{A}$ is concave on $M$.

Let $x \in M \backslash A$ be arbitrary and let $x_{0} \in C L(A)$ be a point with $d\left(x, x_{0}\right)=d(x, C L(A))$. Then $d_{A}\left(x_{0}\right) \geq d_{A}(x)$.

Proof. Assume the contrary, thus $d_{A}\left(x_{0}\right)<d_{A}(x)$. Consider a geodesic $\gamma$ from $x_{0}$ to $x$. Since $h:=d_{A} \circ \gamma$ is concave, the derivative of $h$ at 0 is positive. In particular, $x_{0}$ is non-critical for the function $d_{A}$. Thus, the gradient curve $\eta_{x_{0}}$ of $d_{A}$ starting at $x_{0}$ is non-constant. Moreover, the angle between $\gamma^{\prime}(0)$ and $\nabla_{x_{0}} d_{A}$ is less than $\frac{\pi}{2}$.

By the first variation formula, the derivative of $l(t):=d\left(x, \eta_{x_{0}}(t)\right)$ at 0 is negative. Hence, for small $t>0$,

$$
d\left(x, \eta_{x_{0}}(t)\right)<d\left(x, x_{0}\right) .
$$

Due to Proposition 1.2, $\eta_{x_{0}}(t)$ is contained $C L(A)$. Thus, $x_{0}$ is not a nearest point to $x$ in $C L(A)$. This contradiction finishes the proof.

\subsection{Simple counterexample}

The following example shows that without a completeness assumption no higher smoothness of the distance function can be expected:

Example 4.3. Let $M$ be the flat non-complete manifold $\mathbb{R}^{2} \backslash C$, where $C$ is the ray $C=\{(0, t): t \geq 0\}$. Let $A$ be the singleton $(1,0) \in M$. It is not difficult to see (for instance, using that the completion of $M$ is $C A T(0),[21$, Proposition 12.1], thus uniquely geodesic) that $\operatorname{Reg}^{d_{A}}$ is the whole complement $M \backslash A$. While $M$ is smooth and $A$ is a smooth submanifold, the distance function $f$ to $A$ is not smooth: level sets of $f^{-1}(s)$ of $f$ are concatenations of parts of Euclidean circles of radii s and $s-1$ and thus not $\mathcal{C}^{2}$.

\section{Subsets of positive reach}

\subsection{Characterization}

We are going to provide: 
Proof of Proposition 1.3. Proposition 1.1 shows the equivalence of (1) and (2). Moreover, by Proposition 1.1, (3) implies (2).

It remains to show that (2) implies (3). Thus, let $O$ be a neighborhood of $A$ such that $f=d_{A}$ is $\mathrm{e}^{1,1}$ in $O \backslash A$. Then $f$ is semiconvex on $O \backslash A$ and we need to show that $f$ is semiconvex around any point $x \in A$. Consider a special ball $U_{x}$ around $x$ of radius $r$. Let $W$ be the ball of radius $\frac{r}{3}$ around $x$. For any $q_{1}, q_{2} \in W$ with midpoint $m$ we either have $m \in A$ and then

$$
f(m)=0 \leq \frac{1}{2}\left(f\left(q_{2}\right)+f\left(q_{2}\right)\right) .
$$

Or $m \notin A$. Then the gradient curve $\eta_{m}$ of $f$ starting in $m$ is a geodesic on $\left[0, \frac{r}{3}\right]$. As in the last part of the proof of Proposition 1.1, we deduce

$$
f(m) \leq \frac{1}{2}\left(f\left(q_{1}\right)+f\left(q_{2}\right)\right)+\frac{C}{8} \cdot d^{2}\left(q_{1}, q_{2}\right),
$$

for some $C$ depending only on $U_{x}$. Hence $f$ is semiconvex in $W$, finishing the proof.

\subsection{Geodesics in normal directions}

Now we derive:

Proof of Proposition 1.4. The distance function $f=d_{A}$ is semiconvex in a neighborhood $O$ of $A$. Thus, we have a well-defined (directional) differential $D_{X} f: T_{X} M \rightarrow \mathbb{R}$ and this differential coincides with the distance to the tangent cone $T_{x} A$, cf. [24].

By the definition of normal directions, we have $D_{x} f(h)=1$. In other words, $\left.\left(f \circ \gamma^{h}\right)^{\prime}\right)(0)=1$. By semiconvexity of $f \circ \gamma^{h}$, we have

$$
\lim _{t \rightarrow 0}\left(f \circ \gamma^{h}\right)^{\prime}(t)=1 \text {. }
$$

By the first variation formula, this implies that for $t \rightarrow 0$, the angle between $\left(\gamma^{h}\right)^{\prime}(t)$ and the unique shortest geodesic from $\gamma^{h}(t)$ to $A$ converges to $\pi$. Thus the angle between the gradient curve $\eta^{t}$ of $f$ starting at $\gamma^{h}(t)$ and $\gamma^{h}$ at the point $\gamma^{h}(t)$ converges to 0 . Since, the gradient curves $\eta^{t}$ are geodesics on a fixed interval $\left[0, s_{0}\right]$, we deduce that $\eta^{t}:\left[0, s_{0}\right] \rightarrow O$ converge to $\gamma^{h}$.

Thus, $d\left(\gamma^{h}(t), A\right)=t$ for all $t \in\left[0, s_{0}\right]$. This implies the claim.

\section{Harmonic coordinates and submanifolds}

\subsection{Harmonic and distance coordinates}

We provide:

Proof of Proposition 1.6. Thus, let $M$ be a manifold with two-sided curvature bounds. Let $G: V \rightarrow \mathbb{R}^{n}$ be some distance coordinates on an open subset $V$ of $M$ and let $f: V \rightarrow \mathbb{R}$ be a harmonic function.

We identify $V$ with $G(V) \subset \mathbb{R}^{n}$. The Riemannian metric $g$ of $M$ restricted to is Lipschitz continuous on $V$, [8, Theorem 13.2].

Let $x \in V$ be arbitrary and consider arbitrary harmonic coordinates $F: O \rightarrow \mathbb{R}^{n}$ defined on a neighborhood $O$ of $x$. Thus, the coordinates of $F$ are harmonic functions in $O$.

Due to Nikolaev's theorem, the pull-back Riemannian metric $\tilde{g}:=\left(F^{-1}\right)^{\star}(g)$ is $\mathcal{C}^{1, \alpha}$ on $\tilde{O}:=F(O) \subset \mathbb{R}^{n}$, for all $\alpha<1$, [8, Theorem 14.2]. In particular, $\tilde{g}$ is locally Lipschitz continuous.

Then $F:(O, g) \rightarrow(\tilde{O}, \tilde{g})$ is an isometry between Riemannian manifolds with ${ }^{0,1}$ Riemannian metrics. And such an isometry is always $\mathcal{C}^{1,1}$, [35]. See also [22] and [39] for other proofs of this fact. Hence, $F$ and $F^{-1}$ are of class $\mathrm{e}^{1,1}$.

The function $F^{-1} \circ f$ is harmonic on $\tilde{O}$. By elliptic regularity, [38] or [37, p. 689], the function $F^{-1} \circ f$ is $\mathfrak{C}^{3, \alpha}$. In particular, it is $\mathrm{e}^{1,1}$. Hence, $f=F \circ F^{-1} \circ f$ is $\mathrm{e}^{1,1}$ on $O$ as well. This finishes the proof. 


\subsection{Submanifolds}

This subsection is devoted to

Proof of Proposition 1.7. Let $M$ be a manifold with two-sided bounded curvature and let $N$ be a $e^{1,1}$ submanifold with respect to distance coordinates.

The statement is local. We fix any point $x \in N$ and consider a special neighborhood $U_{x}$ of $x$ in $M$. Then we find a compact $\mathrm{C}^{1,1}$ submanifold $\hat{N}$ of $M$ which is contained in $U_{x}$ and such that $N$ and $\hat{N}$ coincide in a neighborhood of $x$.

Since the statement is local we may assume that $N=\hat{N}$. We may further assume that $U_{x}$ is a distance chart and identify it with a ball in $\mathbb{R}^{n}$. Then the distance in $U$ is given by a Lipschitz continuous Riemannian metric $g$.

By [25, Proposition 1.5, Theorem 1.2], the compact submanifold $N$ has positive reach. Due to [25, Proposition 1.5] and [23, Theorem 1.2], the space $N$ is $C A T(\kappa)$ for some $\kappa$. It thus remains to show that $N$ has curvature bounded from below.

Applying Proposition 1.6 and Nikolaev's approximation theorem, [8, Theorem 15.1], we may further assume on $U$ there exist smooth Riemannian metrics $g^{\epsilon}$, which are uniformly Lipschitz continuous and converge to the Riemannian metric $g$. Moreover, the Riemannian manifold $\left(U, g^{\epsilon}\right)$ have sectional curvature uniformly bounded from above and below.

Smoothing the submanifold $N$ we find a family of smooth submanifolds $N^{\epsilon} \subset U$ with uniform bounds on their $\mathrm{C}^{1,1}$-norms (thus any $N^{\epsilon}$ is a union of a uniform number of charts each of them of bounded $\mathrm{e}^{1,1}$-norm), such that $N^{\epsilon}$ converge to $N$ in $\mathrm{C}^{1}$ sense.

Considering $N^{\epsilon}$ with the the intrinsic metric induced by $g^{\epsilon}$, we see that $N^{\epsilon}$ converge to $N$ in the GromovHausdorff metric (in fact, the convergence is much stronger). Thus, it remains to show that all $N^{\epsilon}$ are Alexandrov space of curvature $\geq-\kappa$, for some fixed $\kappa$. However, the $\mathrm{e}^{1,1}$ bounds of $N^{\epsilon}$ directly imply, that the second fundamental forms of $N^{\epsilon}$ are uniformly bounded (as subsets of the flat space $\mathbb{R}^{n}$ and therefore, of the Riemannian manifold $\left(U, g^{\epsilon}\right)$ ). Applying the Gauß equation, we derive a uniform lower bound on the sectional curvatures of $N^{\epsilon}$. By Toponogov's theorem and compactness of $N^{\epsilon}$, the manifolds $N^{\epsilon}$ are Alexandrov spaces of curvature $\geq-\kappa$. This finishes the proof.

Acknowledgements: The authors are grateful to Anton Petrunin, Carlo Sinestrari and the anonymous referee for helpful comments.

V.K. is partially supported by a Discovery grant from NSERC; A. L. was partially supported by the DFG grants SFB TRR 191 and SPP 2026.

Conflict of interest: Authors state no conflict of interest.

\section{References}

[1] P. Albano, P. Cannarsa, K. T. Nguyen, and C. Sinestrari, Singular gradient flow of the distance function and homotopy equivalence, Math. Ann. 356 (2013), no. 1, 23-43. MR 3038120

[2] L. Ambrosio, N. Gigli, and G. Savaré, Gradient flows in metric spaces and in the space of probability measures, Lectures in Mathematics ETH Zürich, Birkhäuser Verlag, Basel, 2005. MR 2129498

[3] S. Alexander, V. Kapovitch, and A. Petrunin, Alexandrov geometry, Preprint, https://arxiv.org/abs/1903.08539 (2019).

[4] P. Albano, On the cut locus of closed sets, Nonlinear Anal. 125 (2015), 398-405. MR 3373591

[5] B. Andrews, Notes on the isometric embedding problem and the Nash-Moser implicit function theorem, Surveys in analysis and operator theory (Canberra, 2001), Proc. Centre Math. Appl. Austral. Nat. Univ., vol. 40, Austral. Nat. Univ., Canberra, 2002, pp. 157-208. MR 1953483

[6] V. Bangert, Sets with positive reach, Arch. Math. (Basel) 38 (1982), no. 1, 54-57. MR 646321

[7] Y. Burago, M. Gromov, and G. Perelman, A. D. Aleksandrov spaces with curvatures bounded below, Uspekhi Mat. Nauk 47 (1992), no. 2(284), 3-51, 222. MR 1185284

[8] V. N. Berestovskij and I. G. Nikolaev, Multidimensional generalized Riemannian spaces, Geometry, IV, Encyclopaedia Math. Sci., vol. 70, Springer, Berlin, 1993, pp. 165-243, 245-250. MR 1263965 
[9] P. Cannarsa and W. Cheng, Singularities of solutions of Hamilton-Jacobi equations, a rxiv.org/abs/2101.02075, 2021.

[10] P. Cannarsa, W. Cheng, and A. Fathi, Singularities of solutions of time dependent Hamilton-Jacobi equations. applications to Riemannian geometry, arxiv.org/abs/1912.04863, 2019.

[11] P. Cannarsa and C. Sinestrari, Semiconcave functions, Hamilton-Jacobi equations, and optimal control, Progress in Nonlinear Differential Equations and their Applications, vol. 58, Birkhäuser Boston, Inc., Boston, MA, 2004. MR 2041617

[12] D. M. DeTurck and J. L. Kazdan, Some regularity theorems in Riemannian geometry, Ann. Sci. École Norm. Sup. (4) 14 (1981), no. 3, 249-260. MR 644518

[13] H. Federer, Geometric measure theory, Die Grundlehren der mathematischen Wissenschaften, Band 153, Springer-Verlag New York Inc., New York, 1969. MR 0257325

[14] M. Gromov, Metric structures for Riemannian and non-Riemannian spaces, english ed., Modern Birkhäuser Classics, Birkhäuser Boston, Inc., Boston, MA, 2007, Based on the 1981 French original, With appendices by M. Katz, P. Pansu and S. Semmes, Translated from the French by Sean Michael Bates. MR 2307192

[15] M. Ghomi and J. Sprouck, Total curvature and the isoperimetric inequality in Cartan-Hadamard manifolds, Preprint, https://arxiv.org/abs/1908.09814 (2019).

[16] V. Kapovitch, M. Kell, and C. Ketterer, On the structure of RCD spaces with upper curvature bounds, arXiv:1908.07036, 2019.

[17] V. Kapovitch and A. Lytchak, Structure of submetries, Preprint, https://arxiv.org/abs/2007.01325 (2020).

[18] N. Kleinjohann, Nächste Punkte in der Riemannschen Geometrie, Math. Z. 176 (1981), no. 3, 327-344. MR 610214

[19] A. Lytchak and K. Nagano, Geodesically complete spaces with an upper curvature bound, arXiv:1804.05189, 2018.

[20] N. Lebedeva and A. Nepechiy, Alexandrov regions, Preprint (2020).

[21] A. Lytchak and S. Wenger, Isoperimetric characterization of upper curvature bounds, Acta Math. 221 (2018).

[22] A. Lytchak and A. Yaman, On Hölder continuous Riemannian and Finsler metrics, Trans. Amer. Math. Soc. 358 (2006), no. 7 , 2917-2926. MR 2216252

[23] A. Lytchak, On the geometry of subsets of positive reach, Manuscripta Math. 115 (2004), no. 2, 199-205. MR 2098470

[24] A. Lytchak, Open map theorem for metric spaces, Algebra i Analiz 17 (2005), no. 3, 139-159. MR 2167848

[25] A. Lytchak, Almost convex subsets, Geom. Dedicata 115 (2005), 201-218. MR 2180048

[26] C. Mantegazza and A. C. Mennucci, Hamilton-Jacobi equations and distance functions on Riemannian manifolds, Appl. Math. Optim. 47 (2003), no. 1, 1-25. MR 1941909

[27] I. G. Nikolaev, The Synge's formula for geodesic variations in a space of bounded curvature by A. D. Aleksandrov, preprint (Russian), 1988.

[28] _ Bounded curvature closure of the set of compact Riemannian manifolds, Bull. Amer. Math. Soc. (N.S.) 24 (1991), no. 1, 171-177. MR 1056559

[29] Y. Otsu and T. Shioya, The Riemannian structure of Alexandrov spaces, J. Differential Geom. 39 (1994), no. 3, 629-658. MR 1274133

[30] G. Perelman, DC-structures on Alexandrov spaces, preprint, preliminary version (1994).

[31] S. Peters, Convergence of Riemannian manifolds, Compositio Math. 62 (1987), no. 1, 3-16. MR 892147

[32] A. Petrunin, Parallel transportation for Alexandrov space with curvature bounded below, Geom. Funct. Anal. 8 (1998), no. 1, 123-148. MR 1601854

[33] A. Petrunin, Semiconcave functions in Alexandrov's geometry, Surveys in differential geometry. Vol. XI, Surv. Differ. Geom., vol. 11, Int. Press, Somerville, MA, 2007, pp. 137-201. MR 2408266

[34] J. Rataj and L. Zajíček, On the structure of sets with positive reach, Math. Nachr. 290 (2017), no. 11-12, 1806-1829. MR 3683461

[35] I. K. Sabitov, On the smoothness of isometries, Sibirsk. Mat. Zh. 34 (1993), no. 4, 169-176, iv, x. MR 1248802

[36] W.-X. Shi, Deforming the metric on complete Riemannian manifolds, J. Differential Geom. 30 (1989), no. 1, 223-301.

[37] I. H. Sabitov and S. Z. Šefel, Connections between the order of smoothness of a surface and that of its metric, Sibirsk. Mat. Ž. 17 (1976), no. 4, 916-925. MR 0425855

[38] M. E. Taylor, Tools for PDE, Mathematical Surveys and Monographs, vol. 81, American Mathematical Society, Providence, RI, 2000, Pseudodifferential operators, paradifferential operators, and layer potentials. MR 1766415

[39] M. Taylor, Existence and regularity of isometries, Trans. Amer. Math. Soc. 358 (2006), no. 6, 2415-2423. MR 2204038 